\section{A New Pentaoxygenated Xanthone from the Fern Cystopteris fragilis}

\author{
Filippo Imperato \\ Dipartimento di Scienze Chimiche dell'Università \\ di Catania, I-95125 Catania, Italy \\ Z. Naturforsch. 45b, 1603-1604 (1990); \\ received June 1, 1990
}

Cystopteris fragilis, Pteridophyta, Aspleniaceae, 1,6-Dihydroxy-3,5,7-trimethoxyxanthone

A new pentaoxigenated xanthone has been isolated from aerial parts of the fern Cystopteris fragilis. By spectroscopic and chemical methods this compound has been shown to be 1,6-dihydroxy3,5,7-trimethoxyxanthone which is of biosynthetic interest in ferns.

\section{Introduction}

Pharmacological activity (e.g. antipsychotic action [1]) and chemotaxonomic interest of xanthones may explain the increasing interest in these substances in recent years. It has been suggested [2] that Cystopteris fragilis complex is the most interesting biosystematic problem in ferns. Xanthone analysis may be of interest in the study of relationships of the taxa of this complex since previous work on the xanthones and flavonoids of Appalachian Asplenium complex [3] and Asplenium adiantum-nigrum complex [4] has shown that these two groups of ferns provide a classic example of additive inheritance of chemical characters.

Earlier work on the chemical constituents of Cystopteris fragilis has led to the identification of two C-glycosylxanthones (mangiferin and isomangiferin) [5] and four flavonoids based on kaempferol (the 3-O-glucoside, the 3,4'-bis-glucoside, the 3-O-(3"-sulphate)glucoside and the 3-O(6"-sulphate)glucoside [6]. In the present work a pentaoxygenated xanthone has been found for the first time in ferns and it has been shown that this compound (isolated from Cystopteris fragilis Bernh) is 1,6-dihydroxy-3,5,7-trimethoxyxanthone (1) which is a new natural product of biosynthetic interest in ferns.

\section{Materials and Methods}

Air dried aerial parts (50 g) of Cystopteris fragilis Bernh (collected on volcano Etna, Sicily) were homogenized and extracted 3 times with hot $95 \%$ ethanol; evaporation of the solvent in vacuo gave $14 \mathrm{~g}$ of residue. The new xanthone (1) was isolated

Verlag der Zeitschrift für Naturforschung, D-7400 Tübingen 0932-0776/90/1100-1603/\$01.00/0 by preparative paper chromatography on Whatman $3 \mathrm{MM}$ paper in $n$-butanol-acetic acid-water (4:1:5, upper phase) (BAW). The bands, as observed under ultraviolet light with ammonia vapor (colour reactions: yellow to orange in $\mathrm{UV}+\mathrm{NH}_{3}$ ) were cut out, eluted with ethanol, concentrated in vacuo and rechromatographed in $15 \%$ acetic acid (HOAc) and in $n$-butanol-ethanol-water $(4: 1: 2.2)$ (BEW). Further purification was carried out on a silica column chromatography (eluting with benzene) which gave $6 \mathrm{mg}$ of xanthone $1 . R_{f}$ values on Whatman N. 1 paper are: 0.79 in BAW; 0.81 in BEW; 0.17 in $15 \%$ HOAc. $R_{f}$ value on TLC on silica: 0.73 in benzene-methanol $(4: 1)$. UV, MS and ${ }^{1} \mathrm{H}$ NMR data of xanthone $\mathbf{1}$ are shown in Table I.

\section{Complete demethylation of xanthone (1)}

The xanthone $(2 \mathrm{mg})$ and HI $(4 \mathrm{ml} ; \mathrm{d}=1.7)$ were refluxed for $6 \mathrm{~h}$ in the dark. Removal of hydriodic acid under high vacuum gave a residue which was chromatographed. The single spot in TLC on silica (solvent system: benzene-methanol, $4: 1$ ) and on paper chromatography (solvent system: $\mathrm{AcOH}-37 \% \mathrm{HCl}$-water, $30: 3: 10)$ was indistinguishable from a sample of 1,3,5,6,7-pentahydroxyxanthone from Cascora decussata.

\section{Results and Discussion}

Colour reactions and UV spectral analysis in the presence of the customary shift reagents (Table I) suggest that the isolated compound is a xanthone with a free hydroxyl group at position 3 and/or 6 (shift with $\mathrm{NaOAc}$ ) and a free hydroxyl group at position 1 and/or 8 (shift with $\mathrm{AlCl}_{3}$ and $\mathrm{AlCl}_{3}$ ) $\mathrm{HCl}$ ) [7]. The mass spectrum of this compound (Table I) exhibited a molecular ion at $m / z 318$ for $\mathrm{C}_{16} \mathrm{H}_{14} \mathrm{O}_{7}$ in accord with a xanthone containing two hydroxyl groups and three methoxyl groups. The ${ }^{1} \mathrm{H}$ NMR spectrum (Table I) confirmed the presence of these substituents since signals were observed for three methoxyl groups $(\delta 3.89,3.97$ and 4.01) and for two hydroxyl groups $(\delta 12.95$ and 5.08) one of which must be in position 1 or 8 [8]. In addition the position and coupling of two meta-split doublets $(J=2.3 \mathrm{~Hz})$ at $\delta 6.52$ and 6.36 suggested [8] 1,3-dioxygenation in A-ring of the xanthone. A singlet at $\delta 7.42$ was assigned to C-8 proton in spite of the fact that a $\mathrm{C}-8$ proton would give a signal at lower field (between 7.70 and 8.05 [9]) since the signal of this proton is shifted to higher field by the presence of three substituents on the B-ring of the isolated xanthone [8]. This conclusion is confirmed by the ${ }^{1} \mathrm{H}$ NMR spectrum of synthetic 1,3,5,6,7-pentamethoxyxanthone [10] which 
Table I. UV, MS and ${ }^{1} \mathrm{H}$ NMR data of xanthone $\mathbf{1 .}$

\begin{tabular}{|c|c|c|c|c|}
\hline \multicolumn{2}{|c|}{$\mathrm{UV} \lambda_{\max }^{\mathrm{MeOH}}(\mathrm{nm})$} & \multirow{2}{*}{$\frac{\mathrm{MS} m / z \text { (rel. int.) }}{318\left(\mathrm{M}^{+}, 100 \%\right)}$} & \multicolumn{2}{|c|}{${ }^{1} \mathrm{H} \mathrm{NMR}\left(\delta\left(\mathrm{CDCl}_{3}\right), 250 \mathrm{MHz}\right)$} \\
\hline- & $352,315,255$ & & $-\mathrm{OCH}_{3}$ & $3.89,3.97,4.01$ ( $3 \mathrm{H}$ each, $\mathrm{s})$ \\
\hline $\mathrm{AlCl}_{3}$ & $397,345,268$ & $303(8)$ & $6-\mathrm{OH}$ & $5.08(1 \mathrm{H}, \mathrm{s})$ \\
\hline $\mathrm{AlCl}_{3} / \mathrm{HCl}$ & $396,330,265$ & $288(12)$ & $2-\mathrm{H}, 4-\mathrm{H}$ & $6.36,6.52(1 \mathrm{H}$ each, $\mathrm{d}, J=2.3 \mathrm{~Hz})$ \\
\hline $\mathrm{NaOAc}$ & 377.260 & $275(10)$ & $8-\mathrm{H}$ & $7.42(1 \mathrm{H}, \mathrm{s})$ \\
\hline \multirow[t]{5}{*}{$\mathrm{NaOMe}$} & 377,261 & $257(11)$ & $1-\mathrm{OH}$ & $12.95(\mathrm{IH}, \mathrm{s})$ \\
\hline & & $245(3)$ & & \\
\hline & & $229(4)$ & & \\
\hline & & $145(7)$ & & \\
\hline & & $123(5)$ & & \\
\hline
\end{tabular}

shows a singlet at $\delta\left(\mathrm{CDCl}_{3}\right) 7.38$ (C-8 proton); in addition calculations based on the work of Barraclough et al. [8] suggest that $8-\mathrm{H}$ of a xanthone with three hydroxy- and/or methoxy-substituents on the B-ring should appear as singlet a $\delta\left(\mathrm{CDCl}_{3}\right)$ 7.13 whereas $7-\mathrm{H}, 6-\mathrm{H}$ and $5-\mathrm{H}$ of such xanthone are predicted to give a singlet at $\delta\left(\mathrm{CDCl}_{3}\right) 6.10$, 6.46 and 6.25 respectively. The presence of a C-8 proton in the B-ring of the isolated xanthone is confirmed by the absence in the mass spectrum (Table I) of peaks from the loss of $\mathrm{OH}, \mathrm{H}_{2} \mathrm{O}$ and $\mathrm{CHO}$ from the molecular ion since these peaks are typical [11] for xanthones and related compounds with a methoxyl substituent peri to carbonyl group.

The above observations show that the isolated xanthone must be 1,3-dihydroxy-5,6,7-trimethoxyxanthone or 1,6-dihydroxy-3,5,7-trimethoxyxanthone. The presence of a 1,3,5,6,7-oxygenation pattern in the isolated xanthone was confirmed by complete demethylation of this compound with HI which gave 1,3,5,6,7-pentahydroxyxanthone identified by direct comparison with an authentic sample from Cascora decussata [12]. Since the UV spectra of the isolated xanthone in the presence of $\mathrm{NaOAc}$ and $\mathrm{NaOMe}$ are superimposable (Table I), the isolated xanthone seems to be 1,6-dihydroxy-3,5,7-trimethoxyxanthone since the above spectra are not superimposable [7] if a 1,3-dihydroxyl system is present in the xanthone. This conclusion was confirmed by direct comparison of the isolated xanthone (1) with an authentic sample of 1,6-dihydroxy-3,5,7-trimethoxyxanthone which was obtained by selective demethylation [13] of 1,3,5,6,7-pentamethoxyxanthone prepared according to Ghosal et al. [12].<smiles>COc1cc(O)c2c(=O)c3cc(OC)c(O)c(OC)c3oc2c1</smiles>

Xanthone $\mathbf{1}$ is the first pentaoxygenated xanthone isolated from ferns. In 1969 Carpenter et al. [14] predicted that 1,3,5,6,7-oxygenated xanthones should occur in plants if the phenol oxidative coupling biosynthetic mechanism is operative. This suggestion was confirmed in 1974 by Ghosal et al. which isolated [12] for the first time a number of xanthones having the predicted oxygenation pattern from Cascora decussata Schult. In the present work it is shown that a xanthone with the above oxygenation pattern is present also in ferns.

The author thanks the Board of Education (Rome) for financial support.
[1] S. Ghosal, P. V. Sharma, R. K. Chaudhuri, and S. K. Bhattacharya, J. Pharm. Sci. 64, 80 (1975).

[2] J. D. Lovis, Adv. in Bot. Res. 4, 229 (1977).

[3] D. M. Smith and D. A. Levin, Am. J. Bot. 50, 952 (1963).

[4] P. M. Richardson and E. Lorenz-Liburnau, Am. Fern J. 72, 103 (1982).

[5] P. M. Richardson and E. Thaddeus, J. Nat. Prod. 46, 747 (1983)

[6] F. Imperato, Chem. Ind. (London) 1983, 204.

[7] A. A. Lins Mesquita, D. De Barros Correa, O. R. Gottlieb, and M. Taveira Magalhaes, Anal. Chim. Acta 42, 311 (1968).

[8] D. Barraclough, H. D. Locksley, F. Scheinmann,
M. Taveira Magalhaes, and O. R. Gottlieb, J. Chem. Soc. (B) 1970, 603.

[9] K. Hostettmann and H. Wagner, Phytochemistry 16, 821 (1977)

[10] A. J. Quillinan and F. Scheinmann, J. Chem. Soc. Perkin I 1973, 1329.

[11] P. Arends and P. Helboe, Org. Mass Spec. 7, 667 (1973).

[12] S. Ghosal, R. K. Chaudhuri, and K. R. Markham, J. Chem. Soc. Perkin I 1974, 2528.

[13] R. K. Chaudhuri, F. Zymalkowski, and S. Ghosal, J. Phar. Sci. 67, 1321 (1978).

[14] I. Carpenter, H. D. Locksley, and F. Scheinmann, Phytochemistry 8,2013 (1969). 